(C) 1996 IEEE. Personal use of this material is permitted. However, permission to reprint/republish this material

for advertising or promotional purposes or for creating new collective works for resale or redistribution to servers

or lists, or to reuse any copyrighted component of this work in other works must be obtained from the IEEE.

\title{
Integral Dipole Field Calibration of the SRRC Storage Ring Combined Function Bending Magnets
}

\author{
J. C. Lee, Peace Chang and C. S. Hsue* \\ Synchrotron Radiation Research Center
}

\section{Abstract}

Due to the presence of fringe $® e l d$ on both magnet edges, the real trajectory traced out by the beam will deviate from expectation. The integral dipole strength seen by the beam will then have a discrepancy to design value. The beam tracing method is employed to correct fringe $® e l d$ effect and to calibrate integral dipole strength of the bending magnets by tuning magnet current. Final correction factor was achieved in the condition of simulated results meet to design requirements. During machine commissioning, the measured betatron tunes were found different from modeling and a correction factor was applied to correct this tune differences. This factor is in well agreement with that predicted by the beam tracing method.

\section{I: Introduction}

The bending magnet for the SRRC storage ring is a C-type magnet. It is an assembly from lamination iron plates with its pole face tilted to combine dipole $®$ eld and quadrupole $®$ eld. Though the magnetic ®eld has been well measured, the measurement is based on ideal trajectory, curve in bending region and straight for the other section. For the presence of fringe ®eld at magnet edges the real trajectory of the beam would deviate from ideal one. The magnetic ${ }^{\circledR}$ eld seen by the beam will have a little difference due to this trajectory discrepancy. It implies a ®ne calibration is necessary if the difference has signi®cant effect. In this report, beam tracing method is employed to check this difference by taking the eighth mass produced bending magnet as an ensemble. A tracing program is written to simulate the real trajectory of the electron in real magnetic ®eld and the dipole ®eld is calibrated based on this simulated trajectory. Since simulated trajectory dependent on the ®eld the beam encounted, there are several way to get the correction by changing @eld distribution such as shifting, rotating the magnet ${ }^{[1]}$ or changing its power current. However it is very $\operatorname{dif} \circledast$ cult to take into account shifting (rotating) factor in installation. Correction on power current is selected for ®eld adjusting tool.

In this paper, results from tracing prediction are compared with that obtained from commissioning. Algorithm for the beam tracing method is described in section II. Error estimation of the tracing program and the tracing prediction are also given in section II. Commissioning results for this topics can be found in section III. The consistency of these two approaches are quit good.

\section{II: Tracing program and tracing prediction}

The beam tracing program simulates the median plane trajec- tory of the electron and obtains related parameters from the measured magnetic ®eld. Cartesian coordinate system $(\mathrm{X}, \mathrm{Z})$ is used for tracing study with $\mathrm{X}$ the horizontal and $\mathrm{Z}$ the longitudinal coordinate respectively. Origin of the coordinate system is chosen at the magnet center. The motion of the electron in the median plane obeys ${ }^{[2]}$

$$
\frac{X^{\prime \prime}}{\left(1+X^{\prime 2}\right)^{3 / 2}}=-\frac{B}{B_{0} \rho}
$$

in which $\mathrm{X}$ is the horizontal position of tracing point, $B_{0} \rho$ is the ring rigidity and $\mathrm{B}$ the vertical magnetic $®$ eld of tracing point, which is interpolated from measured $®$ eld by three or fourpoint bivriate method ${ }^{[3]}$. Since no signi ${ }^{\circledR}$ cant difference between these two ®eld interpolated method, less than $2 \times 10^{-7}$, threepoint bivriate method is used. The differentiation in equation (1) is w.r.t. the longitudinal. Interpolation from one point to the next point is calculated from equation (1) by Runge-Kutta method. Since rigidity includes the information of beam energy, the program can simulate the trajectory for $® x e d$ energy of electron in the known $® e l d$ distribution or, on the contrary, to get the information of beam energy and simulated trajectory in the ${ }^{\circledR}$ xed $® e l d$ distribution. The former situation is applied in this report while the latter can be applied to measure the beam energy ${ }^{[4]}$.

A testing tracing was $囚$ rst performed to check the accuracy of the tracing program by ideal isomagnet ®eld distribution with design $® e l d$ value and effective length. Error bar of the traced parameters in this testing tracing are all within $0.007 \%$, which is quite enough for the study, and position error for testing tracing point is also within the alignment tolerance. Accuracy of the tracing program would be increased a little bit in real ®eld tracing for it has continuous ®eld distribution instead of hard edge one.

In tracing simulation, the following strategies were considered: a) integral dipole strength (bending angle) within design requirement, b) symmetry simulated trajectory w.r.t. magnet center, c) position error for tracing points outside effective bending within alignment tolerance. Strategy a) ensures the electron beam will be bent to a circle within a tolerable error. Strategies b) and c) push simulated trajectory meeting to ideal trajectory except that in effective bending section. These considerations are very important for the beam diagnostics.

Since $®$ eld measurement extended far away from the magnet, the magnetic ®eld outside the measured area is assumed to zero for the ®eld is neglectable small at this large distance. The tracing step is the same as that used in testing tracing, $5 \mathrm{~mm}$ for far away region and $0.2 \mathrm{~mm}$ for points close to the magnet. Under above conditions, design energy of electron were traced in the ensemble ®eld distribution, which is measured at the median plane of the eighth mass produced bending magnet powered at nominal current. Table 1 lists this uncorrected tracing results. 
Table I

Comparison of the raw data tracing results with the ideal values, in which $B l$ integral strength, $l_{\text {eff }}$ effective length, $\theta$ bending angle, $B_{c}$ center $® e l d$, subindex $s$ indicates tracing starting point, $t$ the magnet entrace point, $e$ the magnet exit point, and $d$ the tracing ending point.

\begin{tabular}{|l|c|c|c|}
\hline & Ideal Value & tracing Results & Error \\
\hline$B l\left(\mathrm{~T}^{*} \mathrm{~m}\right)$ & 1.513666 & 1.521917 & $0.54 \%$ \\
\hline$l_{\text {eff }}(\mathrm{m})$ & 1.22 & 1.222098 & $Đ$ \\
\hline$\theta(\mathrm{deg})$ & 20 & 20.10779 & $0.54 \%$ \\
\hline$B_{c}(\mathrm{~T})$ & 1.24071 & 1.245336 & $Ð$ \\
\hline$x_{s} / z_{s}(\mathrm{~mm})$ & $-912.198 /-106.928$ & $-912.198 /-106.928$ & $Đ$ \\
\hline$x_{t} / z_{t}(\mathrm{~mm})$ & $-606.998 /-53.113$ & $-606.998 /-53.418$ & $Đ$ \\
\hline$x_{e} / z_{e}(\mathrm{~mm})$ & $607.002 /-53.114$ & $607.002 /-54.872$ & $Đ$ \\
\hline$x_{d} / z_{d}(\mathrm{~mm})$ & $911.002 /-106.660$ & $911.002 /-108.789$ & $Đ$ \\
\hline
\end{tabular}

From the data shown in table 1 it is clear that design energy of electron is overbent by about $0.5 \%$ as magnet is powered at nominal current. The $0.5 \%$ overbent has been taken into account the effect from trajectory as well as the contribution of fringe ®eld. It is straightforward to scale down the magnetic ®eld by this amount approximately and design energy of electron is traced again in this scaled down $® e l d$. After some efforts the ®nal correction factor for the magnetic ®eld is $0.575 \%$ instead of $0.5 \%$. This small discrepancy is coming from the magnetic ®eld seen by design energy of electron is slightly changed due to different trajectory followed. Table 2 lists these corrected tracing results.

Table II

Comparison of the corrected tracing results with the ideal values.

\begin{tabular}{|l|c|c|c|}
\hline & Ideal Value & Tracing Results & Error \\
\hline$B l\left(\mathrm{~T}^{*} \mathrm{~m}\right)$ & 1.513666 & 1.513827 & $0.01 \%$ \\
\hline$l_{\text {eff }}(\mathrm{m})$ & 1.22 & 1.223162 & $Đ$ \\
\hline$\theta(\mathrm{deg})$ & 20 & 20.00821 & $0.04 \%$ \\
\hline$B_{c}(\mathrm{~T})$ & 1.24071 & 1.237635 & $Đ$ \\
\hline$x_{s} / z_{s}(\mathrm{~mm})$ & $-912.198 /-106.928$ & $-912.198 /-106.928$ & $Đ$ \\
\hline$x_{t} / z_{t}(\mathrm{~mm})$ & $-606.998 /-53.113$ & $-606.998 /-53.417$ & $Đ$ \\
\hline$x_{e} / z_{e}(\mathrm{~mm})$ & $607.002 /-53.114$ & $607.002 /-53.583$ & $Đ$ \\
\hline$x_{d} / z_{d}(\mathrm{~mm})$ & $911.002 /-106.660$ & $911.002 /-106.958$ & $Đ$ \\
\hline
\end{tabular}

Correction on the magnetic @eld is achieved by tuning its power current. The necessity of $0.575 \%$ reduction on the magnetic ${ }^{\circledR}$ eld also reveals nominal current is too large for design energy of electron. From the good $®$ eld uniformity and the linear relation between the $®$ eld and its power current within this small range, ${ }^{\circledR}$ nal correction factor on the current is also predicted by $0.575 \%$.

The tracing results listed in table 2 are in good agreement with the design except magnet center $®$ eld and effective length. These discrepancies are coming from simulated trajectory deviated from ideal. As we known the main function of the bending magnet is to bend design energy of electron with the desired angle. Hence bending angle and integrated dipole strength are the most important parameters to be considered. The error of integrated dipole strength (bending angle) is within $4 \times 10^{-4}$, which is well within the speci®cation of $1 \times 10^{-3}$. From above simu- lated results it is clear that integral dipole strength has been well calibrated from reducing the nominal current by $0.575 \%$.

\section{III: Experience in the storage ring commissioning}

In storage ring commissioning, the measured tunes are found not consistent with modeling. The tune difference makes the application program, which is based on modeling, don't work well. With the study and correction of the tune differences, a model closing to real machine is gotten. The tunes of machine are then measured under different lattices and compared with that predicted by modeling, as shown in table 3 .

Table III

Tune Differences between measurement and modeling before correction.

\begin{tabular}{|c|c|c|c|}
\hline Lattice & measured $\nu_{x} / \nu_{y}$ & modeling $\nu_{x} / \nu_{y}$ & $\Delta \nu_{x} / \Delta \nu_{y}$ \\
\hline $\mathrm{A}$ & $7.353 / 4.080$ & $7.4448 / 4.1133$ & $0.0918 / 0.0333$ \\
\hline $\mathrm{B}$ & $7.232 / 4.080$ & $7.3220 / 4.1151$ & $0.0900 / 0.0351$ \\
\hline $\mathrm{C}$ & $7.236 / 4.080$ & $7.3210 / 4.1120$ & $0.0850 / 0.0320$ \\
\hline $\mathrm{D}$ & $7.206 / 4.092$ & $7.2927 / 4.1272$ & $0.0867 / 0.0352$ \\
\hline $\mathrm{F}$ & $7.210 / 4.087$ & $7.2949 / 4.1186$ & $0.0849 / 0.0316$ \\
\hline $\mathrm{G}$ & $7.227 / 4.091$ & $7.3147 / 4.1228$ & $0.0877 / 0.0318$ \\
\hline $\mathrm{H}$ & $7.221 / 4.086$ & $7.3078 / 4.1169$ & $0.0868 / 0.0309$ \\
\hline $\mathrm{I}$ & $7.235 / 4.082$ & $7.3245 / 4.1121$ & $0.0895 / 0.0301$ \\
\hline
\end{tabular}

The contribution of individual quadrupole in tunes are also investigated by the formula

$$
\delta \nu=\frac{1}{4 \pi} \int k_{1} \beta d s \simeq \frac{\bar{\beta}}{4 \pi} \frac{1}{f}
$$

with $\bar{\beta}$ is averaged $\beta$ function and $\frac{1}{f}=k_{1} l$ the inverse of focus length, and results of one super-period in the 6-fold symmetry lattice are shown in table 4.

Table IV

Estimated $\delta \nu_{x} / \delta \nu_{y}$ in one super period with $k_{1}$ strength (T/m), $l$ length (m), and $\beta_{x} / \beta_{y}$ beta functions (m).

\begin{tabular}{rcccccc}
\hline \hline Name & $k_{1}$ & $l$ & $\overline{\beta_{x}}$ & $\overline{\beta_{y}}$ & $\delta \nu_{x}$ & $\delta \nu_{y}$ \\
\hline \hline & & & & & & \\
$Q_{1}$ & -1.50815 & 0.35 & 12.3 & 5.9 & -0.51189 & +0.24783 \\
$Q_{2}$ & 2.87048 & 0.35 & 18.8 & 4.6 & +1.50304 & -0.36777 \\
$Q_{3}$ & -1.15592 & 0.24 & 6.2 & 9.9 & -0.13687 & +0.21857 \\
$D M_{1}$ & -0.37 & 1.22 & 1.2 & 10.81 & -0.04311 & +0.38831 \\
$Q_{4}$ & 2.73087 & 0.35 & 6.3 & 2.5 & +0.47318 & -0.19015 \\
$D M_{2}$ & -0.37 & 1.22 & 1.82 & 5.71 & -0.06538 & +0.20511 \\
$Q_{4}$ & 2.73087 & 0.35 & 6.3 & 2.5 & +0.47318 & -0.19015 \\
$D M_{1}$ & -0.37 & 1.22 & 1.2 & 10.81 & -0.04311 & +0.38831 \\
$Q_{3}$ & -1.15592 & 0.24 & 6.2 & 9.9 & -0.13687 & +0.21857 \\
$Q_{2}$ & 2.87048 & 0.35 & 18.8 & 4.6 & +1.50304 & -0.36777 \\
$Q_{1}$ & -1.50815 & 0.35 & 12.3 & 5.9 & -0.51189 & +0.24783 \\
& & & & & & \\
\hline \hline
\end{tabular}

If we add up all of the contribution of quadrupole elements in tunes in table 4, ratio for the summation of horizontal tune to the vertical is around 3.1434, which is very close to the difference tune ratio for individual element in table 3 . This fact indicates the 
discrepancy in tunes between real machine and modeling could come from one source, by which all of the quadrupole strength in the ring can be scaled up or down. The beam energy, which is used as a normalization factor in modeling program, is suspected to produce this scaling factor. If the beam energy increased by a factor of $0.5 \%$ then the tune difference between measurement and modeling is reduced to reasonable level, as shown in table 5 .

Table V

Tune differences between measurement and modeling with correction factor 0.995 .

\begin{tabular}{|c|c|c|c|}
\hline Lattice & measured $\nu_{x} / \nu_{y}$ & modeling $\nu_{x} / \nu_{y}$ & $\Delta \nu_{x} / \Delta \nu_{y}$ \\
\hline $0715-00$ & $7.230 / 4.082$ & $7.2449 / 4.0793$ & $0.0149 /-0.0027$ \\
\hline $0715-01$ & $7.208 / 4.092$ & $7.2220 / 4.0913$ & $0.0120 /-0.0007$ \\
\hline $0715-02$ & $7.244 / 4.079$ & $7.2619 / 4.0750$ & $0.0179 /-0.0040$ \\
\hline $0715-03$ & $7.226 / 4.088$ & $7.2410 / 4.0864$ & $0.0150 /-0.0016$ \\
\hline $0715-04$ & $7.235 / 4.080$ & $7.2514 / 4.0770$ & $0.0164 /-0.0030$ \\
\hline $0715-05$ & $7.261 / 4.081$ & $7.2778 / 4.0787$ & $0.0168 /-0.0023$ \\
\hline $0715-06$ & $7.251 / 4.086$ & $7.2666 / 4.0850$ & $0.0156 /-0.0010$ \\
\hline $0715-07$ & $7.276 / 4.078$ & $7.2946 / 4.0743$ & $0.0186 /-0.0037$ \\
\hline $0715-08$ & $7.258 / 4.088$ & $7.2736 / 4.0857$ & $0.0156 /-0.0023$ \\
\hline $0715-09$ & $7.267 / 4.080$ & $7.2841 / 4.0762$ & $0.0171 /-0.0038$ \\
\hline $0715-10$ & $7.203 / 4.083$ & $7.2127 / 4.0798$ & $0.0097 /-0.0032$ \\
\hline $0715-11$ & $7.193 / 4.088$ & $7.2012 / 4.0858$ & $0.0082 /-0.0022$ \\
\hline $0715-12$ & $7.218 / 4.079$ & $7.2301 / 4.0756$ & $0.0121 /-0.0034$ \\
\hline $0715-13$ & $7.200 / 4.089$ & $7.2086 / 4.0868$ & $0.0086 /-0.0022$ \\
\hline $0715-14$ & $7.208 / 4.080$ & $7.2191 / 4.0774$ & $0.00111 /-0.0026$ \\
\hline $0715-15$ & $7.174 / 4.083$ & $7.1807 / 4.0803$ & $0.0067 /-0.0027$ \\
\hline $0715-16$ & $7.295 / 4.080$ & $7.3106 / 4.0781$ & $0.0156 /-0.0019$ \\
\hline
\end{tabular}

This indicates the integral dipole strength is too strong for design energy of electron and need to be reduced. After some efforts, (®nal scaling factor is 0.99448 . That means the correction factor in dipole ${ }^{\circledR e l d}$ and its powered current is $0.552 \%$, which is in good agreement with that predicts by the tracing simulation.

\section{IV: Conclusion}

The beam tracing method is a good tool to calibrate the magnetic dipole ®eld w.r.t. its powered current as well as for the study of beam energy and its possible tracing trajectory in the known ®eld distribution. In above study design energy of electron is used to calibrate the magnetic @eld and its powered current by beam tracing method. Design energy of electron is found to be overbent by the dipole at its nominal current setting. The excessive factor for integral dipole strength is around $0.5 \%$. As dipole current is reduced by $0.575 \%$, a good results was found with a symmetry trajectory w.r.t. magnet center and position error outside the magnet within alignment tolerance. Integral dipole strength and bending angle are also well within the spec$\mathrm{i} \AA$ cation after this reduction. From storage ring commissioning a tune discrepancy between real machine and model prediction was found. This discrepancy ®nally is indicated to be coming from magnetic dipole $®$ eld is too strong. As the magnet powered current is reduced by $0.552 \%$ the discrepancy in tunes is canceled. This fact shows the good consistency of these two approaches, tracing prediction and the commissioning verication.

In this paper the linear relation between dipole ${ }^{\circledR e l d}$ and its powered current is assumed. Within a small range of variation this linear assumption is a very good approximation. While as the deviation is too much the linear assumption is not hold again and the tracing study need to be performed at this deviated ®eld distribution to give precise prediction.

Since SRRC bending magnet is a combined function one, correction on the dipole ®eld will slightly change the combined quadrupole ®eld. While the correction algorithm for bending magnet is to correct the dipole $®$ eld $®$ rst. Due to the good tunability of the chosen lattice, error of the combined quadrupole $® e l d$ in bending magnet is easier to be overcame by triplet quadrupoles outside the achromate.

\section{Acknowledgement}

The authors would like to thank the magnet measurement group for providing the $®$ eld data.

\section{Reference}

[1] J. C. Lee, C. C. Kuo and C. S. Hsue, Beam Tracing of the SRRC First Prototype Bending magnet, SRRC/BD/IM/8914.

[2] K. Steffen, Basic Course on Accelerator Optics, CERN 85$19,1985$.

[3] M. Abramouitz and I. A. Stegn, Handbook of Mathematical from U.S. Dept. of Commerce, NBS $\underline{55}, 1964$.

[4] M. H. Wang and J. C. Lee, The Algorithm of Energy Measurement in BTS Transport Line, these proceedings. 